\title{
Incidencia estimada de las cardiopatías congénitas en niños menores de 1 año en el Perú
}

\author{
Adriel Olórtegui ${ }^{1,2}$, Manuel Adrianzén ${ }^{3}$
}

Resumen

Palabras clave

\begin{abstract}
Las cardiopatías congénitas han cobrado importancia en las últimas décadas, no solo por su relevancia clínica sino también por su incremento como problema de salud pública. En nuestro país, pese a los avances en el manejo médico, no se ha realizado estudios que determinen la importancia epidemiológica de este grupo de problemas. Objetivo: Determinar la importancia epidemiológica de las cardiopatías congénitas. Diseño: Sistematización de estudios epidemiológicos. Lugar: Departamento de Medicina Preventiva y Salud Pública, Facultad de Medicina de San Fernando, UNMSM. Materiales: Estudios epidemiológicos e información demográfica. Procedimiento: Mediante una recopilación bibliográfica de estudios epidemiológicos e información demográfica, se realizó estimaciones de la incidencia de casos en nuestro país, para el quinquenio 2006-2010. Principales medidas de resultados: Cardiopatías congénitas. Resultados: Se estima que en el 2006 existieron 3925 cardiopatías, de las cuales 83,5\% fueron no cianóticas. Las cifras serían similares anualmente hasta el 2010. También, se estima que representarían el 2,5\% del total de razón de años de vida potencialmente perdidos por cada mil habitantes. Conclusiones: Las cardiopatías congénitas son un problema de salud pública que debe ser tomada en cuenta para preparar a los servicios nacionales de salud. Asimismo, debe emprenderse estudios epidemiológicos sobre este problema.
\end{abstract}

Cardiopatías congénitas; salud pública; incidencia; métodos epidemiológicos.

\begin{abstract}
Estimated prevalence of congenital cardiopaties in one year-old children in Peru Abstract

The importance of congenital cardiopaties has changed in the last decades, not only due to their clinical relevance but also as it is considered a problem of public health. In our country, in spite of the advances in medical care, no studies have been done to determine the epidemilogical relevance of these deseases . Objective: To determine the epidemiologic relevance of congenital heart diseases in Peru. Design: Systematizing of epidemic studies. Setting: Department of Preventive Medicine and Public Health, Faculty of Medicine San Fernando, UNMSM. Materials: Epidemiologic studies and demographic information. Proceeding: Based on bibliographical recopilation of epidemiologic studies and demographic information, we estimated the incidence of congenital cardiopaties cases in our country for 2006-2010. Main outcome measures: Congenital cardiopaties. Results: For 2006,
\end{abstract}

1 Profesor Auxiliar del Departamento de Medicina Preventiva y Salud Pública. Facultad de Medicina Humana, UNMSM. Lima, Perú.

2 Coordinador del Área de Epidemiología. Instituto Nacional del Corazón, EsSalud. Lima, Perú.

3 Jefe del Departamento de Cardiopediatría. Instituto Nacional del Corazón, EsSalud. Lima, Perú.
3925 cardiopaties were determined, 83,5\% of them non cianotic; this prevalence would be similar annually up to 2010 and they would represent 2,5\% of potentially life rate years lost per 1000 inhabitants. Conclusions: Congenital cardiopaties represent a public health problem that should be considered by the national healthcare system; also, epidemiologic studies should beundertaken.

Key words: Heart defects, congenital; public health; incidence; epidemiologic methods.

\section{INTRODUCCIÓN}

Desde que, en 1939, Robert Gross realizó la primera intervención quirúrgica de un paciente con cardiopatía congénita (CC) $\left({ }^{1}\right)$, éstas han cobrado gran importancia para la medicina y la salud pública. Entre las características que denotan su importancia se identifica el incremento de su incidencia en las últimas décadas $\left({ }^{2}\right)$, ser las malformaciones congénitas más frecuentes y el progreso alcanzado en el diagnóstico y tratamiento de estos problemas $\left(^{3}\right)$. 
Numerosos estudios han establecido que la incidencia de las cardiopatías congénitas es de aproximadamente $0,8 \%$, con un rango que va de 0,4 a $1,2 \%(1,4,5)$, variaciones que dependen del momento del estudio, población de estudio y métodos diagnósticos. Por ejemplo, se ha encontrado una incidencia mayor en los estudios realizados desde la incorporación del diagnóstico ecocardiográfico, así como cuando se ha estudiado series de recién nacidos muertos. Estos resultados han ido de la mano con los avances en el tratamiento y consiguiente mejora de la supervivencia de estos pacientes, lo muestra la prevalencia de las cardiopatías congénitas en los menores de 16 años, que se encuentra en $1,2 \%\left({ }^{6}\right)$,

A raíz de los progresos evidenciados en el tratamiento de las cardiopatías congénitas, los servicios de salud han ido adecuándose y desarrollando estructuras y procesos para el manejo de estos pacientes, lo que resulta en altos costos para cualquier sistema de salud. En nuestro país, tanto el Ministerio de Salud (MINSA) como EsSalud (los dos principales y mayores prestadores de servicios de salud del país) han abordado el problema; el primero a través de su Seguro Integral de Salud (SIS) cubre el tratamiento de los niños con estos problemas $\left.{ }^{7}\right)$ y el segundo con la creación del Departamento de Cardiopediatría del Instituto Nacional del Corazón (INCOR) $\left(^{8}\right)$. Cabe precisar que la atención para estos pacientes debe cubrir desde el diagnóstico hasta la rehabilitación y su implementación requiere establecer la cantidad de recursos y servicios necesarios, tales como cardiólogos, cardiopediatras, servicios de ecocardiografía, cardiología intervencionista, cirujanos cardiopediátricos así como centros quirúrgicos y servicios de rehabilitación con profesionales idóneos.

En este sentido, resulta necesario conocer las características epidemiológicas de las cardiopatías congénitas, a fin de determinar cuántos cardiólogos, cardiopediatras, servicios asistenciales y demás recursos son necesarios para afrontar adecuadamente la atención que requieren estas afecciones. Consecuentemente, conocer su magnitud, distribución y perfil clínico-epidemiológico permitirá planificar las acciones que deberán tomarse para brindar asistencia a los pacientes afectados y disminuir su impacto en la mortalidad, discapacidad y carga social.

No existen en el país estudios de base poblacional o epidemiológicos que permitan una aproximación al conocimiento de la magnitud y distribución por tipo de cardiopatías congénitas, por lo que el objetivo de este estudio es estimar en forma indirecta la frecuencia de este grupo de malformaciones para el lustro 2006 a 2010, a partir de la información publicada en estudios epidemiológicos y también mediante el uso de la información demográfica del país. Complementariamente, se describirá la distribución estimada de los tipos de cardiopatías congénitas.

Más allá de la utilidad de este trabajo, el propósito es alertar acerca de la importancia de las cardiopatías congénitas en nuestro país, así como impulsar al desarrollo de investigaciones que permitan un conocimiento más exacto y directo de nuestra realidad en este campo.

\section{MATERIALES Y MÉTODOS}

Las estimaciones obtenidas en el presente trabajo han sido realizadas a partir de las tasas de incidencia publicadas en diferentes estudios en el ámbito mundial, conjuntamente con los datos demográficos del Perú. La metodología ha tenido tres etapas, la primera de revisión documental, la segunda de sistematización y selección de los valores base para las estimaciones y tercera, el cálculo de las estimaciones respectivas. 
La revisión documental tuvo por objetivo recuperar bibliografía sobre estudios epidemiológicos de incidencia de las cardiopatías congénitas, así como la recuperación de información demográfica del Perú. La búsqueda se realizó a través del PubMed y buscadores de la internet, procediéndose a la recuperación de las publicaciones mediante el acceso a las bases de datos bibliográficas y páginas web respectivas. Los trabajos publicados obtenidos fueron un total de 12 estudios epidemiológicos, con información relacionada a los objetivos del estudio y un tratado de cardiología pediátrica de la Sociedad Española de Cardiología, de donde se ha tomado la información correspondiente para el cálculo de las estimaciones.

La información demográfica se obtuvo a través del portal web del Instituto Nacional de Estadística e Informática (INEI). Se tomó los resultados de los X Censos Nacionales $\left({ }^{9}\right)$ $\mathrm{y}$ documentos técnicos sobre proyecciones demográficas para realizar los cálculos proyectados para el quinquenio 2006-2010 $\left({ }^{10,11}\right)$. Con la información referida se procedió a determinar la población menor de un año (denominador de las estimaciones).

En la segunda etapa se procedió a la revisión de las publicaciones sobre la incidencia de cardiopatías congénitas, para seleccionar los valores reportados y utilizarlos en los cálculos de la tercera etapa. Se seleccionó aquellos estudios de base poblacional, sea estos a partir de estudios longitudinales específicos o de registros continuos que mantienen algunos países o ciudades; se tomó como valores para las estimaciones las tasas medianas y las variaciones comunicadas. Para establecer las distribuciones según tipo de $\mathrm{CC}$, se procedió a tomar los valores relativos, sea en tasas o porcentaje, lo que permitió establecer las 10 cardiopatías más frecuentes así como la distribución relativa de cada grupo de cardiopatías congénitas.
Para la tercera etapa, con los valores seleccionados se procedió al cálculo de los estimados de incidencia en el país, mediante el siguiente proceso:

1. De las estimaciones demográficas obtenidas se procedió a tomar la población proyectada de menores de 1 año.

2. De los estudios sobre incidencia, se tomó las tasas menores, medianas y mayores.

3. Al valor obtenido de población menor de un año, se le aplicó el valor de las tasas seleccionadas y se procedió a calcular el número estimado de casos con su rango respectivo de valores menores y mayores esperados.

El procedimiento descrito permitió establecer el rango estimado de casos de cardiopatía congénita que ocurriría en el Perú y que se presenta a continuación.

\section{RESULTADOS}

Sobre la base de la información obtenida, se presenta el resumen sistemático de la epidemiología de las cardiopatías congénitas, las proyecciones de población para menores de un año y la incidencia estimada de las cardiopatías para el quinquenio indicado.

\section{Incidencia de cardiopatías congénitas}

Las cardiopatías congénitas representan la tercera parte de todas las malformaciones congénitas, con una incidencia muy variable, considerando los valores comunicados en diversos estudios, que oscilan entre $4 \mathrm{y}$ 50 por mil nacidos vivos $\left({ }^{12}\right)$. El valor de incidencia mayormente señalado en los estudios es de 8 por mil, el cual es aceptado como valor de referencia $\left({ }^{2,5,6,13,14}\right)$. Dentro de los tipos de cardiopatía congénita, las acianóticas son las más frecuentes, pues representan aproximadamente el $83 \%$ de to- 
das las cardiopatías congénitas, mientras que las cianóticas agrupan el $17 \%$, con una incidencia de 5,4 por mil para las primeras y de 1.3 por mil para el segundo grupo $\left(^{2}\right)$.

Aproximadamente, entre 80 y $90 \%$ de todas las cardiopatías congénitas están agrupadas en 10 tipos específicos $\left(^{5}\right)$, los cuales, aunque con ligeras variaciones según los estudios, corresponden a los siguientes: comunicación interventricular, persistencia del ducto arterioso, comunicación interauricular, estenosis pulmonar valvular, tetralogía de Fallot, coartación de la aorta, canal auriculoventricular completo, transposición de los grandes vasos, estenosis aórtica y ventrículo izquierdo hipoplásico. Algunos autores han incluido entre este grupo al tronco arterioso común, la atresia tricuspídea y la doble salida del ventrículo derecho $\left({ }^{2,5,15,16}\right)$. La incidencia y frecuencia relativa de estas cardiopatías se muestra en la Tabla 1.

De las 10 primeras cardiopatías congénitas, seis corresponden a las acianóticas y las restantes cuatro a las cianóticas. Igualmente, el grado de severidad es variable en seis de las cardiopatías (van de leves o moderadas a severas), mientras que las restantes son consideradas cuadros severos. Entre las cardiopatías congénitas no cianóticas, las más frecuentes son los defectos específicos del septo cardíaco: comunicación interventricular (CIV) y comunicación interauricular (CIA), las que en conjunto representan aproximadamente el $50 \%$ de este grupo y el $35 \%$ de todas las cardiopatías. Por su parte, la cardiopatía congénita cianótica más frecuente es la tetralogía de Fallot, que representa el $70 \%$ de este grupo y alrededor del $10 \%$ de este grupo de afecciones.

Consideradas en forma individual, la CIV, CIA, PDA y comunicación auri- culoventricular (CAV) son las cardiopatías congénitas más frecuentes, lo que coloca a los problemas del septo cardiaco como el área anatómica cardiaca más afectada. Luego, se ubican los problemas valvulares y las cardiopatías complejas, considerando entre las diez más frecuentes a la EP, la TF, la estenosis aórtica congénita (EAC); finalmente, los problemas de los vasos y las cámaras cardiacas representan problemas importantes, tales como la coartación de la aorta (CoAo), la transposición de los grandes vasos (TGV) y el ventrículo izquierdo hipoplásico (VIH).

Las tasas presentadas en la Tabla 1 son representativas de la incidencia mundial de las cardiopatías congénitas y se las utilizará para realizar las estimaciones de la incidencia, toda vez que la principal fuente considerada es la revisión realizada por Hoffman y col, que han incluido 55 estudios epidemiológicos sobre la incidencia de las cardiopatías $\left({ }^{2}\right)$. Más aun, estudios posteriores realizados en países como Brasil, India, España y Chile, han mostrado resultados similares $(5,13,16,17)$.

\section{Información demográfica}

Según los resultados del X Censo Nacional del Población del Perú - 2005, la población menor de un año es de 511 576, la cual representa el $1,96 \%$ del total nacional $\left({ }^{9}\right)$. Sobre la base de esta población y utilizando las proyecciones establecidas por el INEI, se estableció la distribución de menores de 1 año hasta el 2010, que puede verse en la Tabla $2\left({ }^{11}\right)$, seguirá superando el medio millón.

\section{Estimación de las cardiopatías congénitas a nivel nacional ${ }^{*}$}

Con la información epidemiológica presentada y la información demográfica pro-

En la descripción de los resultados se utilizará el término 'media' para referir el empleo de la tasa de incidencia mediana hallada en la bibliografia; se emplea en este sentido para hacer referencia a que se trata de un valor de tendencia central y que en este caso tiene mayor validez que el promedio (medida que se afecta por valores extremos). 
Tabla 1. Características clínico-epidemiológicas de las cardiopatías congénitas más frecuentes*

\begin{tabular}{|c|c|c|c|c|c|c|c|}
\hline \multirow[t]{2}{*}{$\begin{array}{l}\text { Cardiopatía } \\
\text { congénita }\end{array}$} & \multirow[t]{2}{*}{ Orden } & \multicolumn{3}{|c|}{ 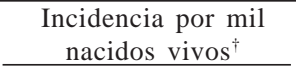 } & \multirow{2}{*}{$\begin{array}{c}\text { Porcentaje } \\
\text { entre } \\
\text { las } \mathrm{CC}^{\ddagger}\end{array}$} & \multirow{2}{*}{$\begin{array}{c}\text { Tipo de } \\
\text { cardiopatía } \\
\text { y severidad }\end{array}$} & \multirow[t]{2}{*}{ Evolución pronóstico } \\
\hline & & Q1 & Mediana & Q3 & & & \\
\hline Todas las cardiopatías & & 6,02 & 7,67 & 10,57 & & & \\
\hline No cianóticas & & & 6,40 & & 83 & & \\
\hline Cianóticas & & 1,08 & 1,27 & 1,53 & 17 & & \\
\hline $\begin{array}{l}\text { Comunicación } \\
\text { interventricular } \\
\text { (CIV) }\end{array}$ & $1^{\circ}$ & 1,76 & 2,83 & 4,48 & 20 & $\begin{array}{l}\text { Leve a severa } \\
\text { No cianótica (las } \\
\text { formas graves sí pueden } \\
\text { cursar con cianosis) }\end{array}$ & $\begin{array}{l}\text { - Hasta el } 90 \% \text { se cierra antes de los } 2 \text { años } \\
\text { · Las grandes y moderadas pueden producir } \\
\text { HTP (hipertensión pulmonar), IC (insufi- } \\
\text { ciencia cardiaca) e infecciones pulmonares }\end{array}$ \\
\hline $\begin{array}{l}\text { Persistencia del } \\
\text { ducto arterioso } \\
\text { (PCA) }\end{array}$ & $2^{\circ}$ & 0,37 & 0,56 & 1,06 & 10 & $\begin{array}{l}\text { No cianótica } \\
\text { Moderada a severa } \\
\text { (pueden causar cianosis } \\
\text { si son severas) }\end{array}$ & $\begin{array}{l}\text { El } 35 \% \text { cierra en el primer mes de vida, } \\
\text { el } 75 \% \text { a los tres meses y casi todos } \\
\text { al año de vida. }\end{array}$ \\
\hline $\begin{array}{l}\text { Comunicación } \\
\text { interauricular } \\
\text { (CIA) }\end{array}$ & $3^{\circ}$ & 0,32 & 0,57 & 0,78 & $10-17$ & $\begin{array}{l}\text { No cianótica } \\
\text { Leve a moderada }\end{array}$ & $\begin{array}{l}\text { - Se desconoce cuántos cierran, si se trata } \\
\text { de ostium secundum. } \\
\text { - Manifestaciones clínicas pueden iniciarse } \\
\text { en la segunda década de la vida }\left({ }^{3}\right) \text {. }\end{array}$ \\
\hline $\begin{array}{l}\text { Estenosis } \\
\text { pulmonar valvular } \\
\text { (EP) }\end{array}$ & $4^{\circ}$ & 0,36 & 0,53 & 0,84 & $8-10$ & $\begin{array}{l}\text { No cianótica } \\
\text { Moderada a severa (en } \\
\text { lesiones severas puede } \\
\text { causar cianosis) }\end{array}$ & $\begin{array}{l}\text { Sin cirugía, el pronóstico es malo; } \\
\text { la valvuloplastía da resultados } \\
\text { excelentes. }\end{array}$ \\
\hline $\begin{array}{l}\text { Tetralogía } \\
\text { de Fallot } \\
\text { (TF) }\end{array}$ & $5^{\circ}$ & 0,29 & 0,36 & 0,58 & $\begin{array}{c}11-13 \\
(70)\end{array}$ & $\begin{array}{l}\text { Cianótica } \\
\text { severa }\end{array}$ & $\begin{array}{l}\text { - Sobreviven } 90 \% \text { a los } 20 \text { años de edad } \\
\text { y } 85 \% \text { a los } 30 \text { años de edad. } \\
\text { · } 5-10 \% \text { de los casos con TF corregidos son } \\
\text { re-operados } 15 \text { por lesiones residuales, a lo } \\
\text { largo de } 20-30 \text { años de seguimiento. } \\
\text { · El tratamiento quirúrgico logra buena } \\
\text { calidad de vida. Mortalidad hospitalaria }<2 \%\end{array}$ \\
\hline $\begin{array}{l}\text { Coartación } \\
\text { de la aorta } \\
\text { (CoAo) }\end{array}$ & $6^{\circ}$ & 0,29 & 0,36 & 0,49 & $\begin{array}{c}4 \\
(3-10)\end{array}$ & $\begin{array}{l}\text { No cianótica } \\
\text { Moderada a severa } \\
\text { (las severas pueden } \\
\text { causar cianosis) }\end{array}$ & $\begin{array}{l}\text { - Supervivencia superior al } 95 \% \text { al año y de } \\
90 \% \text { a los } 10 \text { años. } \\
\text { · El } 20 \% \text { fallece en la } 2^{\text {a }} \text { década de la vida y } \\
\text { el } 85 \% \text {, antes de los } 30 \text { años. } \\
\text { · Asintomáticos hasta los } 15 \text { años. Un } 10 \% \\
\text { desarrolla IC leve y puede sobrevivir sin } \\
\text { intervención hasta la edad adulta. }\end{array}$ \\
\hline $\begin{array}{l}\text { Comunicación } \\
\text { auriculoventricular } \\
\text { (CAV) }\end{array}$ & $7^{\circ}$ & 0,24 & 0,34 & 0,40 & $3-5$ & $\begin{array}{l}\text { Cianótica } \\
\text { Severa }\end{array}$ & $\begin{array}{l}\text { - Sin cirugía, el } 90 \% \text { de la variedad completa } \\
\text { fallece antes de los } 2 \text { años. } \\
\text { · Si sobreviven al primer año, pueden llegar } \\
\text { a la segunda década de la vida. }\end{array}$ \\
\hline $\begin{array}{l}\text { Transposición de } \\
\text { los grandes vasos } \\
\text { (TGV) }\end{array}$ & $8^{\circ}$ & 0,23 & 0,30 & 0,39 & $\begin{array}{l}7-8 \\
(5)\end{array}$ & $\begin{array}{l}\text { Cianótica } \\
\text { Severa }\end{array}$ & $\begin{array}{l}\text { La TGV pura es incompatible con la vida. } \\
\text { - } 60 \% \text { fallece al primer mes y el } 90 \% \\
\text { al primer año. }\end{array}$ \\
\hline $\begin{array}{l}\text { Estenosis aórtica } \\
\text { congénita } \\
\text { (EAC) }\end{array}$ & $9^{\circ}$ & 0,16 & 0,26 & 0,39 & $3-6$ & $\begin{array}{l}\text { No cianótica, moderada } \\
\text { severa (las severas } \\
\text { pueden causar cianosis) }\end{array}$ & $\begin{array}{l}\text { El } 20 \% \text { continua con estenosis leve a los } \\
30 \text { años y un } 20 \% \text { requerirá de válvula. } \\
\text { En la estenosis moderada, } 40 \% \text { requerirá } \\
\text { cirugía con supervivencia del } 81 \% \text { a los } \\
25 \text { años. } \\
\text { - Muerte súbita en el } 2 \% \text { antes de los } 15 \text { años } \\
\text { y el } 29 \% \text { después de los } 15 \text { años. }\end{array}$ \\
\hline $\begin{array}{l}\text { Ventrículo } \\
\text { izquierdo } \\
\text { hipoplásico } \\
\text { (VIH) }\end{array}$ & $10^{\circ}$ & 0,15 & 0,23 & 0,28 & 2,5 & $\begin{array}{l}\text { Cianótica } \\
\text { Severa }\end{array}$ & $\begin{array}{l}\text { Es la mayor causa de muerte de los recién } \\
\text { nacidos en la primera semana de vida ( } 25 \% \\
\text { de todas las muertes de origen cardiaco en } \\
\text { los lactantes). } \\
\text { - La expectativa de vida de estos pacientes } \\
\text { ha ido mejorando en los últimos años } \\
\text { ( } 70 \% \text { de supervivencia a } 5 \text { años). }\end{array}$ \\
\hline
\end{tabular}

\footnotetext{
* Elaboración referencias: 2,4,5,6,12,13,14,16,18,19,20,21,22,23.
}

† Ref. 2. Para las estimaciones, se ha tomado como valores referenciales los valores del cuartil inferior 25\% (Q1), de la mediana 50\% (Q2) y del cuartil superior $75 \%(\mathrm{Q} 3)$.

₹ Los valores que aparecen entre paréntesis y en segunda línea muestran la proporción que representa la CC respectiva dentro del grupo de las CC cianóticas. 
Adriel Olórtegui y col.

Tabla 2. Población nacional y de menores de 1 año. X Censo Nacional de Población del Perú. INEI - 2005.

\begin{tabular}{|c|c|c|c|c|c|c|}
\hline Población & 2005 & 2006 & 2007 & 2008 & 2009 & 2010 \\
\hline Nacional & 26,152265 & 26,518 397 & 26,889654 & 27,266 109 & 27,647835 & 28,007257 \\
\hline Menores 1 año & 511576 & 511805 & 510903 & 509876 & 508720 & 506931 \\
\hline
\end{tabular}

yectada, en la Tabla 3 se muestra la incidencia estimada de casos de cardiopatías congénitas a nivel nacional. Se presenta el rango y valor central de casos, basados en las incidencias del cuartil menor, la mediana y el cuartil superior, proyectadas hasta el 2010. Se presenta las estimaciones para todas las cardiopatías, así como desagregadas por grandes grupos (cianóticas y acianóticas) y las específicas más frecuentes.

Como resultado de la metodología aplicada, se ha podido establecer que durante el quinquenio 2006-2010, el número total de casos de cardiopatías congénitas oscilará en un valor medio entre 3888 y 3925 , con una mayor incidencia de las cardiopatías congénitas acianóticas, que variarán entre 3276 y 3 244. Las cardiopatías cianóticas presentarán un rango entre los 644 y 650 casos (Figura 1).

La distribución según tipo de cardiopatías congénita también muestra un rango de variación muy estrecho de casos estimados. Puede observarse que la cardiopatía más frecuente, la CIV, oscilará entre $\operatorname{los} 1448$ y 1434 casos, seguido de lejos por la PDA, con un rango entre los 290 y 287 casos. El resto de cardiopatías congénitas específicas también presenta rangos de variación estrechos, existiendo casos como las últimas cinco cardiopatías donde el rango de casos cambia entre uno a tres casos para la incidencia media estimada para el periodo 2006 - 2010. Estos resultados tienen tales características debido a

Tabla 3. Casos estimados de cardiopatías congénitas según grupos y tipos de cardiopatía. Perú 2006-2010.

\begin{tabular}{|c|c|c|c|c|c|c|}
\hline \multirow[t]{2}{*}{ Cardiopatías congénitas } & \multicolumn{3}{|c|}{2006} & \multicolumn{3}{|c|}{2010} \\
\hline & Q1 & Q2 & Q3 & Q1 & Q2 & Q3 \\
\hline Todas las cardiopatías & 3081 & 3925 & 5408 & 3052 & 3888 & 5357 \\
\hline No cianóticas & & 3276 & & & 3244 & \\
\hline Cianóticas & 552 & 650 & 785 & 546 & 644 & 777 \\
\hline Comunicación interventricular (CIV) & 899 & 1448 & 2294 & 891 & 1434 & 2272 \\
\hline Persistencia del ducto arterioso (PCA) & 166 & 290 & 400 & 164 & 287 & 396 \\
\hline Comunicación interauricular (CIA) & 190 & 289 & 295 & 189 & 286 & 292 \\
\hline Estenosis pulmonar valvular (EP) & 182 & 272 & 428 & 180 & 270 & 424 \\
\hline Tetralogía de Fallot (TF) & 149 & 182 & 295 & 148 & 180 & 292 \\
\hline Coartación de la aorta (CoAo) & 148 & 182 & 252 & 147 & 180 & 249 \\
\hline Comunicación auriculoventricular (CAV) & 124 & 174 & 203 & 123 & 172 & 201 \\
\hline Transposición de los grandes vasos (TGV) & 118 & 155 & 199 & 117 & 154 & 197 \\
\hline Estenosis aórtica congénita (EAC) & 82 & 131 & 199 & 82 & 130 & 197 \\
\hline Ventrículo izquierdo hipoplásico (VIH) & 79 & 116 & 143 & 78 & 115 & 141 \\
\hline
\end{tabular}




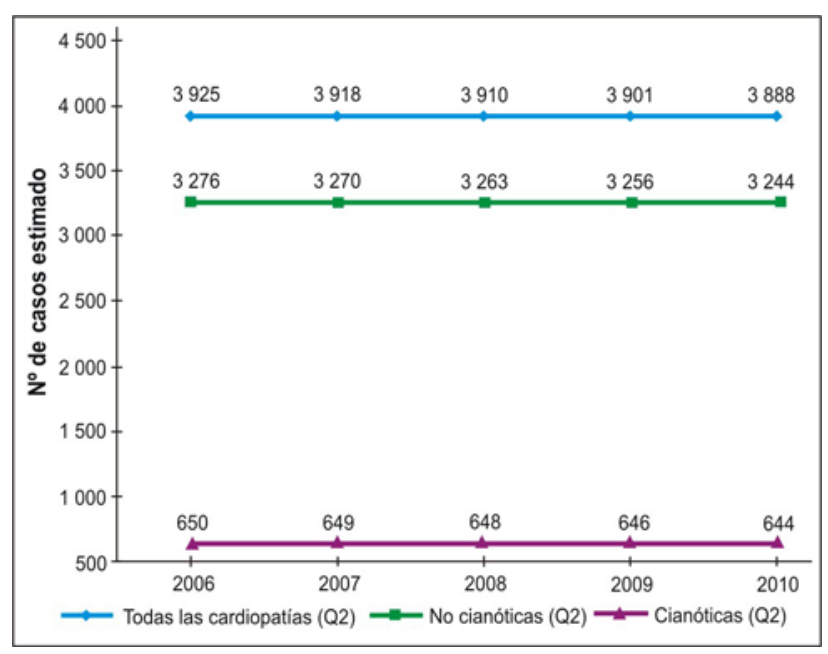

Figura 1. Estimación del número de cardiopatías congénitas según grandes grupos. 2006-2010. Perú.

que los valores base de población y tasas de incidencia utilizadas son fijas, por lo que solo se presenta los valores del 2006 y del 2010 (ver Tabla 3).

Considerando que la aparición de los problemas de salud es variable, se ha estimado también el rango de casos que pueden presentarse en el quinquenio utilizando las incidencias de los cuartiles mayor y menor que se muestran en la Tabla 1 y que son aplicadas para la estimación de casos de las cardiopatías específicas. En este sentido, el mínimo de cardiopatías en el año 2010 sería de 3052 y el máximo de 5357 casos. Entre las cianóticas, el mínimo sería de 546 y el máximo de 777 casos. Para el grupo de cardiopatías acianóticas, no ha sido posible establecer un rango por falta de datos específicos (ver Tabla 3).

En este sentido, para el año 2010, la CIV, como cardiopatía más frecuente, tendría un número que oscila entre los 891 y 2272 casos, mientras que el VIH (décima más frecuente) presentaría un rango entre 78 y 141 casos. El resto de cardiopatías presentaría comportamientos similares dentro del rango de incidencias adoptado para reali- zar el cálculo de las estimaciones (ver Tabla 3 ).

Puede observarse que, para las cardiopatías congénitas de menor incidencia, el rango del número de casos es pequeño y las diferencias entre ellas también.

En resumen, las cardiopatías congénitas afectan a miles de recién nacidos, existe un predominio de las cardiopatías congénitas acianóticas frente a las cianóticas. Además, excluyendo las CIV, la estimación de casos para las cardiopatías más frecuentes no supera los mil casos por año para cada tipo específico. Las implicancias de estos resultados son comentados en el siguiente acápite.

\section{DISCUSIÓN}

Los resultados obtenidos tienen una serie de implicancias importantes para los servicios nacionales de salud, considerando que las cardiopatías congénitas trascienden en lo epidemiológico, en la respuesta de los servicios de salud y en lo social. En lo epidemiológico, si consideramos que casi $1 \%$ de los recién nacidos del país presentan algún tipo de cardiopatía, las cardiopatías congénitas se convierten en un problema de salud pública, no solo por la magnitud del problema sino también por la necesidad de prevención y control que requieren. La respuesta de los servicios de salud va de la mano con la magnitud epidemiológica del problema. En este sentido, es conocido que los infantes portadores de cardiopatías requieren de atención especializada de alto costo y nuestro sistema de servicios de salud no está necesariamente en capacidad de afrontar y que, ante la necesidad de atención de miles de niños con estos problemas, existe la posibilidad de no cubrirla. Finalmente, el impacto social del problema, aunque poco explorado, no deja de ser importante por las 
implicancias estructurales, funcionales y económicas que se producen en las familias de los niños.

En los siguientes acápites se realiza un análisis de las implicancias que la magnitud estimada puede tener en los aspectos indicados.

\section{Aspectos epidemiológicos}

En la Tabla 1, se ha resumido las principales características clínico-epidemiológicas de las cardiopatías congénitas, lo cual sirve de base para describir algunos aspectos de la importancia que, en cuanto a mortalidad, carga de enfermedad y recurrencia, tienen este grupo de enfermedades cardiopediátricas. Un análisis de estas características aplicada a las estimaciones establecidas, permite inferir que las tres cardiopatías más frecuentes no representarían mayor problema epidemiológico.

Las CIV, según diversos textos de cardiología pediátrica, se resuelven espontáneamente. Las cifras estiman que hasta $90 \%$ de los casos se resuelve antes de los dos años y que por lo tanto no requieren de atención $\left({ }^{6,18}\right)$. Tomando estas cifras y considerando la incidencia mediana estimada se tendría que, de los 1448 casos esperados, 1350 se estarían resolviendo sin requerir atención, por lo que alrededor de 150 casos si la requerirían, aunque no necesariamente en el primer año de vida. Situación similar se reporta para el caso de la PDA, por lo que el número de casos que requerirían atención bordearía los 25 casos.

Situación análoga se presentaría con la CIA, puesto que la mayoría de los casos llegan a edad adulta sin presentar manifestaciones o complicaciones clínicas; es decir, alrededor de $80 \%$ requeriría atención recién en la edad adulta $\left({ }^{19}\right)$. Este comportamiento implica que un número mínimo de casos, tal vez 20 en el caso de las estima- ciones de nuestro país, requerirían atención en los primeros años de vida.

Este escenario determina que de la incidencia media, estimada en 2027 casos para las tres cardiopatías congénitas más frecuentes en el año 2006, solo el 10\% (aproximadamente 200 casos) requerirían atención a nivel nacional. Cabe precisar que estas tres primeras cardiopatías representan el $51 \%$ de todas las cardiopatías estimadas.

El análisis anterior permite vislumbrar que alrededor de 1900 casos presentarían cardiopatías de mayor severidad o complejidad, que requerirían atención especializada, de las cuales el $64 \%$ (aproximadamente 1200 casos) correspondería a las otras siete cardiopatías congénitas más frecuentes (desde la EP hasta el VIH de la Tabla 3), muchas de las cuales son incompatibles con la vida.

Entre las cardiopatías incompatibles con la vida y de alta mortalidad pese al tratamiento, se encuentran la TGV, el VIH, la CAV completa y la TF. Para el primer caso, según estudios, el $90 \%$ fallece al primer año $\left({ }^{20}\right)$, lo que determina que al año fallezcan alrededor de 140 lactantes. La mortalidad en el VIH también es elevada, pues el $30 \%$ fallece antes de los cinco años y es responsable del $25 \%$ de las defunciones de causa cardiaca en los recién nacidos $\left({ }^{21}\right)$. Si se toma en forma estimada que el $15 \%$ fallece al primer año, tendríamos que el VIH ocasiona 30 defunciones en menores de 1 año.

La CAV completa sin tratamiento tiene una mortalidad de $90 \%$ a los dos años y la mortalidad luego del tratamiento es de alrededor del $10 \%\left({ }^{22}\right)$, lo que estaría indicando que esta afección produce alrededor de 20 muertes anuales. Para el último tipo de cardiopatía compleja que analizaremos, la $\mathrm{TF}$, es sabido que el tratamiento actual ofrece una supervivencia mayor al $95 \%$, con buena calidad de vida $\left.{ }^{(23}\right)$, lo que se obser- 
va en países donde la cobertura de la asistencia es óptima, no siendo el caso del Perú, donde muchos de los casos con TF no acceden a atención alguna. Cuando no se brinda atención oportuna y adecuada a los pacientes con Fallot, la mortalidad es elevada y alcanza el $80 \%$ al primer año de vida; por lo que, si se considera que la mitad de estos casos no accede a atención en nuestro país, se produciría una mortalidad de 90 casos al año.

El somero análisis que se ha descrito, debe ser observado con mayor relevancia en el futuro, considerando que, como viene ocurriendo en el Perú, conforme disminuye la mortalidad infantil debida a causas prevenibles (principalmente infecciosas), la importancia de otras causas, como las cardiopatías congénitas, se incrementa.

Otro aspecto a considerar es el relativo a la presencia de otras anomalías congénitas, conjuntamente con las cardiopatías congénitas. Según Grech, el $17 \%$ de los niños con cardiopatía tienen alguna otra anomalía congénita, destacando el síndrome de Down como el más frecuente $\left({ }^{24}\right)$. Este aspecto, conlleva uno extra que debe ser abordado, cual es la posibilidad de recurrencia y la necesidad de consejería clínica y genética a los padres de niños con estos problemas.

En resumen, desde una perspectiva clínico-epidemiológica, las cardiopatías congénitas representan un importante problema de salud en la población menor de 1 año, no solo por su magnitud sino también por la carga de enfermedad que representan (ver breve análisis más adelante), además de la demanda de atención especializada que requieren, cuyos aspectos específicos se analiza a continuación.

\section{Aspectos de servicios de salud}

El panorama brevemente descrito configura un escenario en el cual los servicios de salud deben desarrollar capacidades para realizar prevención, diagnóstico precoz, atención curativa oportuna, seguimiento, consejería y rehabilitación. Afrontar este contexto implica que los sistemas y las organizaciones de salud deben contar con servicios asistenciales especializados y de vanguardia. Desde la perspectiva de salud pública y servicios de salud, las estimaciones presentadas deben servir, como acota Subirana $\left({ }^{3}\right)$, para la determinación de las necesidades y planificar su implementación.

En este sentido, los dos sistemas de servicios con que cuenta el país ya han iniciado medidas para afrontar el problema. El MINSA, a través del Seguro Integral de Salud, brinda el financiamiento para el tratamiento de las cardiopatías congénitas; lo cual habilita al Instituto de Salud del Niño y otros establecimientos de salud del país a brindar tratamiento a los niños afectados por este problema $\left({ }^{7}\right)$; además, el Instituto realiza campañas de tratamiento para los niños de mayor gravedad.

Por su parte, el INCOR, a través de su Departamento de Cardiopediatría, ofrece atención especializada desde el año 1998, brindando en la actualidad atención quirúrgica de alta complejidad y resolviendo las cardiopatías tributarias de intervencionismo cardiaco. Según análisis preliminares realizados, el Departamento de Cardiopediatría atiende alrededor de 300 pacientes al año $\left({ }^{25}\right)$.

Sin embargo, estos esfuerzos resultarían insuficientes por cuanto el manejo de este tipo de pacientes requiere de prestaciones integrales que abarcan el seguimiento, monitoreo, rehabilitación y retratamientos. Tal vez, sería menester integrar el esfuerzo de ambas instituciones para afrontar el problema, lo cual redundaría en una disminución de la mortalidad y un mejor pronóstico y calidad de vida de los niños afectados. 
Finalmente, un aspecto que deben abordar los servicios de salud conjuntamente con las instituciones académicas es el desarrollo de investigaciones para el planteamiento de estrategias de prevención y control de las cardiopatías congénitas.

\section{Aspectos sociales}

Más allá de su importancia en el campo de la salud y sus servicios, las cardiopatías congénitas conllevan una serie de efectos sociales, los cuales no han sido suficientemente abordados, pero que deben ser afrontados. Con el propósito de describirlos, conviene considerar los efectos a nivel social, familiar y personal. No abordamos el aspecto económico, porque la atención de este grupo de pacientes no debe ser considerada un gasto sino parte del costo de vivir en sociedad, tomando en cuenta que toda sociedad debe, por un principio de solidaridad, brindar ayuda a los que lo requieren y los niños con cardiopatías no escapan a esta premisa.

Desde la perspectiva social, a partir de un ensayo básico de AVPP (años de vida potencialmente perdidos), estableceremos una aproximación de la carga de morbilidad que una sociedad afronta por las cardiopatías congénitas. En este sentido, si consideramos que un tercio de las cardiopatías fallece $\left({ }^{6}\right)$, tendríamos aproximadamente 1300 defunciones en menores de 1 año en el país, por lo que tomando en cuenta una RAVPP (razón de años de vida potencialmente perdidos (se expresa multiplicado por $10^{\mathrm{K}}$ habitantes)) para las cardiopatías de 3,22 AVPP por mil habitantes, las cardiopatías congénitas el 2,5\% aproximadamente del valor a nivel nacional de referencia como valor la RAVPP nacional del año $2002\left({ }^{26}\right)$.

En el nivel familiar, la presencia de un niño con cardiopatía genera una serie de tribulaciones y temores. Los padres tienen que afrontar por un lado la atención y cura- ción de su hijo (la cual muchas veces no es completa) y en el caso de las parejas jóvenes, el temor de procrear un producto con el mismo defecto u otro aún mayor (situación que según diferentes estudios puede repetirse entre un 10 y $30 \%$ ) $\left(^{6}\right)$. Adicionalmente, debe considerarse el gasto familiar para el tratamiento del niño con cardiopatía, el cual varía según tenga o no cobertura de atención. En el primer caso, el gasto se reduce al traslado del niño y familiares y gastos complementarios (compra de utensilios para higiene, por ejemplo); mientras que, en el segundo caso, debe agregarse medicamentos, análisis, etc., gastos que no están al alcance de muchas economías familiares. Más aún, debe considerarse que el manejo de los niños con cardiopatías congénitas se extiende más allá del contacto con los servicios de salud.

No pretendemos agotar el análisis de la trascendencia que tendrían las cardiopatías congénitas en nuestro país, pero sí establecer claramente la necesidad de estudiarlas desde una perspectiva epidemiológica, cuyos resultados permitirán plantear un abordaje más eficiente y de mayor calidad en el manejo de este grupo de anomalías congénitas.

\section{Conclusiones}

Las cardiopatías congénitas son un problema de gran importancia, no solo por su trascendencia clínica reflejada en la especialización y complejidad de manejo que requieren, sino también desde la perspectiva de la salud pública, si se considera la importancia que tienen dentro de la mortalidad infantil y que previsiblemente se incrementará conforme se controlen más las causas prevenibles de mortalidad en este grupo de edad.

No existen estudios epidemiológicos de base poblacional acerca de la magnitud o riesgo de las cardiopatías en el país, por lo 
que resulta necesario iniciar su desarrollo. En este sentido, una alianza que involucre al sistema de servicios nacionales de salud, la comunidad médica, la universidad y sociedades científicas podría integrar esfuerzos y desarrollar un registro nacional para el desarrollo de investigaciones epidemiológicas.

Las estimaciones presentadas muestran que los afectados por estos problemas podrían ascender a algunos miles de niños, la mayoría de los cuales carecen de acceso a los servicios con capacidad para resolver el problema y también por la poca alerta o tecnología existente en los servicios de salud para detectarlos, lo que conllevaría a que un número considerable de niños fallezca o se desarrolle con limitaciones, que podrían ser resueltas si se contara con las condiciones adecuadas. Huelga reiterar que el conocimiento epidemiológico generado localmente, referido no solo a la ocurrencia sino también a la distribución geográfica y socioeconómica, permitiría atender y evitar defunciones y mejorar la calidad de vida de estos niños.

\section{REFERENCIAS BIBLIOGRÁFICAS}

1. Hoffman JI, Kaplan S, Liberthson RR. Prevalence of congenital heart disease. Am Heart J. 2004;147(3):425-39.

2. Hoffman JI, Kaplan S. The incidence of congenital heart disease. J Am Coll Cardiol. 2002;39(12):1890-900.

3. Subirana MT. Cardiopatías congénitas: presente y futuro. Rev Esp Cardiol. 2005;58(12):1381-4.

4. Harris JA, Francannet C, Pradat P, Robert E. The epidemiology of cardiovascular defects, part 2: a study based on data from three large registries of congenital malformations. Pediatr Cardiol. 2003;24(3):222-35.

5. Martínez OP, Romero IC, Alzina de Aguilar V. Incidencia de las cardiopatías congénitas en Navarra (1989-1998). Rev Esp Cardiol. 2005;58(12):1428-34.

6. Moreno G F. Epidemiología de las Cardiopatías Congénitas. [monografía en Internet]. En: Asociación Española de Pediatría. Protocolos de la Sociedad Española de Cardiología Pediátrica. Madrid: AEP; 2005 [acceso 30 de octubre de 2006]. Disponible en: http://www.aeped.es/protocolos/ protocolos secp.htm
7. Ministerio de Salud. SIS oficializa tratamientos de hemodiálisis, neoplasias, leucemias y cardiopatías congénitas [monografía en Internet]. Lima: MINSA; 2004 [acceso 15 de marzo de 2007]. Disponible en: http://www.minsa.gob.pe/ocom/ prensa/notadeprensa.asp?np codigo $=1134 \&$ mes $=2 \&$ anio $=$ 2004

8. EsSalud. Reglamento de Organización y funciones del Instituto Nacional del Corazón-INCOR. Resolución de Gerencia General. Lima: EsSalud; 1997.

9. Instituto Nacional de Estadística e Informática. Censos Nacionales 2005: X de Población y V de Vivienda [monografía en Internet]. Lima: INEI; 2007 [acceso 15 de marzo de 2007]. Disponible en: http://iinei.inei.gob.pe/iinei/ cpv2005/index.asp

10. Instituto Nacional de Estadística e Informática. Perú: Estimaciones y Proyecciones de población total, urbana y rural por años calendarios y edades simples, 1970-2025. Lima: INEI; 2002. Report No. 15.

11. Instituto Nacional de Estadística e Informática. Nuevas proyecciones nacionales de población del Perú por departamentos urbano y rural y sexo, 2005 a 2020. Lima: INEI; 2006.

12. Viñals LF, Giulliano BA. Cardiopatías congénitas. Incidencia postnatal. Rev Chil Obstet Ginecol. 2002;67:203-6.

13. Dos Santos GJ. Epidemiological characteristics of congenital heart diseases in Londrina, Parana south Brazil. Arq Bras Cardiol. 2000;74(5):395-404.

14. Pradat P, Francannet C, Harris JA, Robert E. The epidemiology of cardiovascular defects, part I: a study based on data from three large registries of congenital malformations. Pediatr Cardiol. 2003;24(3):195-221.

15. Garne E. Congenital heart defects - occurrence, surgery and prognosis in a Danish County. Scand Cardiovasc J. 2004;38(6):357-62.

16. Miyague NI, Cardoso SM, Meyer F, Ultramari FT, Araujo FH, Rozkowisk I, et al. Epidemiological study of congenital heart defects in children and adolescents. Analysis of 4,538 cases. Arq Bras Cardiol. 2003;80(3):269-78.

17. Viñals LF, Giulliano BA. Cardiopatías congénitas. Incidencia postnatal (II). Rev Chil Obstet Ginecol. 2002;67(3):20710.

18. Torres O. Cardiopatías congénitas. En: Montoya M, editor. Cardiología. $6^{\mathrm{a}}$ ed. Medellín: Corporación para Investigaciones Biológicas; 2003. p. 138-86.

19. Conejo L. Defectos septales auriculares [monografía en Internet]. En: Protocolos Diagnósticos y Terapéuticos en Cardiología Pediátrica: Sevilla: Sociedad Española de Cardiología Pediátrica y Cardiopatías Congénitas; 2005 [acceso 15 de marzo de 2007]. Disponible en: http:// www.secardioped.org/protocolos/protocolos/ Capitulo 4.pdf

20. Gil-Gournier M, Álvarez A. Transposición de los grandes vasos [monografía en Internet]. En: Protocolos Diagnósticos y Terapéuticos en Cardiología Pediátrica: Sevilla: Sociedad Española de Cardiología Pediátrica y Cardiopatías Congénitas; 
2005 [acceso 15 de marzo de 2007]. Disponible en: http:// www.secardioped.org/protocolos/protocolos/Capitulo 13.pdf

21. Zunzunegui J. Síndrome del corazón izquierdo Hipoplásico [monografía en Internet]. En: Protocolos Diagnósticos y Terapéuticos en Cardiología Pediátrica: Sevilla: Sociedad Española de Cardiología Pediátrica y Cardiopatías Congénitas; 2005 [acceso 15 de marzo de 2007]. Disponible en: http:// www.secardioped.org/protocolos/protocolos/Capitulo_17.pdf

22. Casaldáliga J. Defectos de los cojines endocárdicos [monografía en Internet]. En: Protocolos Diagnósticos y Terapéuticos en Cardiología Pediátrica: Sevilla: Sociedad Española de Cardiología Pediátrica y Cardiopatías Congénitas; 2005 [acceso 15 de marzo de 2007]. Disponible en: http:// www.secardioped.org/protocolos/protocolos/Capitulo 6.pdf

23. Rodríguez M, Villagrá F. Tetralogía de Fallot [monografía en Internet]. En: Protocolos Diagnósticos y Terapéuticos en Cardiología Pediátrica: Sevilla: Sociedad Española de Cardiología Pediátrica y Cardiopatías Congénitas; 2005 [acceso 15 de marzo de 2007]. Disponible en: http:// www.secardioped.org/protocolos/protocolos/Capitulo 11.pdf
24. Grech V, Gatt M. Syndromes and malformations associated with congenital heart disease in a population-based study. Int J Cardiol. 1999;68(2):151-56.

25. Olórtegui A. Análisis de la hospitalización del INCOR EsSalud. Enero 2000 - Junio 2006. Lima: EsSalud; 2006.

26. Dirección General de Epidemiología. Análisis de la Situación de Salud del Perú - 2005. Lima: MINSA; 2006.

Manuscrito recibido el 18 de junio de 2007 y aceptado para publicación el 20 de agosto de 2007.

\section{Correspondencia:}

Adriel Olórtegui Yzú

Departamento de Medicina Preventiva y Salud Pública

Facultad de Medicina, UNMSM.

Av. Grau 750. Lima 1, Perú.

Correo-e: aolortegui@unmsm.edu.pe 